\title{
Versalzt BENEFIT die Salzhypothese bei MS?
}

Fragestellung: Besteht eine Assoziation zwischen der Menge der Kochsalzaufnahme und der Konversion zur Multiplen Sklerose (MS) bei Patienten mit klinisch isoliertem Syndrom (KIS)?

Hintergrund: In-vitro-Experimente und Tierversuche zeigten, dass eine kochsalzreiche Diät verstärkt zu proinflammatorischen Th17-Zellen und dadurch zu einem aggressiveren Verlauf einer experimentellen Autoimmunenzephalitis (EAE) führt. Die vermehrte Salzzufuhr erhöhte die Expression der Serum- und glukokortikoidinduzierbaren Proteinkinase 1 (SGK1), die unter anderem den Natriumpegel in Zellen reguliert. SGK1 führte zu einer vermehrten Expression des Interleukin(IL)-23-Rezeptors, wodurch die Differenzierung von T-Zellen zu proinflam-matorischen Th17-Zellen gefördert wird. Außerdem wurde durch die SGK1 das „forkhead box protein O1“ (FOXO1), das die Expression des IL-23-Rezeptors negativ reguliert, supprimiert. Passend dazu verminderte Kochsalz in der EAE die suppressive Wirkung von regulatorischen T-Zellen und die Anzahl antiinflam-

Fitzgerald KC, Munger KL, Hartung HP et al. Sodium intake and multiple sclerosis activity and progression in BENEFIT. Ann Neurol 2017; 82: 20 -9 matorischer Makrophagen.

Eine 2-Jahres-Studie mit 70 Probanden zeigte erstmalig einen Zusammenhang zwischen Salzausscheidung im Urin als Korrelat der quantitativen Salzzufuhr und der Krankheitsaktivität (Schubrate, neue T2-Läsionen) bei Patienten mit MS [1]. Jedoch ergaben zwei pädiatrische MS-Studien keine Assoziation zwischen der Natriumaufnahme und der Schubrate oder dem Risiko, eine MS zu entwickeln [2, 3].

Patienten und Methodik: Analysiert wurden Urinproben von 468 Probanden mit KIS, die an der BENEFIT-Studie teilgenommen hatten. In einem Beobachtungszeitraum von fünf Jahren wurde die Konversion zur MS gemäß Poser- als auch den 2001 revidierten McDonald-Kriterien untersucht.

Ergebnisse: Im Median wurden pro Proband 14 Urinproben gesammelt. Die geschätzte Natriumausscheidung lag bei täglich 3,7 g. Bei $39 \%(n=150)$ und $75 \%(n=217)$ der Probanden wurde innerhalb der Studiendauer die Diagnose MS gestellt. Es bestand keine Assoziation zwischen der geschätzten täglichen $\mathrm{Na}$ triumausscheidung und der Zeit bis zur Konversion in eine MS, der Schubrate, der Zeit bis zum ersten Schub nach Konversion, zur EDSS-Progression sowie der MRT-Aktivität.

Schlussfolgerung: Die Autoren folgern, dass die Menge der Kochsalzaufnahme nicht im Zusammenhang mit der MSKrankheitsaktivität steht.

\section{- Kommentar von Refik Pul und Christoph Kleinschnitz, Essen}

\section{Die Salzhypothese bleibt weiterhin ungeklärt}

Diese Ergebnisse stellen die Humanrelevanz der Salzhypothese bei MS infrage. Wie könnte man die Diskrepanz zwischen Tierversuch und Patientenstudie erklären? In der Schlüsselstudie der Erlanger Kollegen wurde das Futter der Mäuse mit 4\% und ihr Wasser mit $1 \%$ Kochsalz angereichert [4]. Eine $20 \mathrm{~g}$ schwere Maus hat demnach bei maximaler Nahrungs- und Trinkmenge eine tägliche Kochsalzaufnahme von zirka $15 \mathrm{mg} / \mathrm{g}$ Körpergewicht (KG) unter der Berücksichtigung, dass Mäuse etwa 3-6 g Nahrung und 4-7 ml Wasser zu sich nehmen. Der tägliche Natriumkonsum in Deutschland liegt bei Männern bei 3,2 g und bei Frauen bei 2,4 g. Ein zirka $70 \mathrm{~kg}$ schwerer Mann nimmt also eine Natriummenge von zirka 0,05 $\mathrm{mg} / \mathrm{g} \mathrm{KG/Tag}$ auf und müsste täglich die 300-fache Menge zu sich nehmen, um die Bedingungen der Labormäuse zu erfüllen.

In der BENEFIT-Studie wurden die Einzelurinausscheidungen, aus denen $90 \%$ der täglichen Kochsalzaufnahme geschätzt werden kann, longitudinal untersucht. Da die ermittelten Werte aber eine hohe intraindividuelle Streuung zeigten, nahmen die Autoren eine statistische Glättung vor. Betrachtet wurden also geglättete "Langzeitnatriumwerte" und Patienten mit erhöhter Natriumausscheidung oder Spitzen in der Natriumaufnahme (z. B. Männer mit höherem BMI) blieben unberücksichtigt. Die Bedingungen in der aktuellen Patientenstudie und in den Tierversuchen sind also nicht vergleichbar. Auf Populati- onsebene gibt es für die Salzhypothese derzeit noch kein Indiz. Die aktuelle Arbeit kann die Salzhypothese nicht widerlegen, denn eine Analyse von MS-Patienten mit wirklich hohem Salzkonsum (> $6 \mathrm{~g}$ Natrium pro Tag) erfolgte nicht. Unabhängig von diesem Ergebnis und zur Vorbeugung von kardiovaskulären Erkrankungen würden MS-Patienten von einer Empfehlung zur moderaten Salzrestriktion aber ohnehin profitieren.

\footnotetext{
Referenzen

1. Farez MF et al. J Neurol Neurosurg Psychiatry 2015; 86: 26-31

2. McDonald J et al. Mult Scler Relat Disord 2016; 6: 87-92

3. Nourbakhsh B et al. J Neurol Neurosurg Psychiatry 2016; 87: 1350 - 3

4. Kleinewietfeld M et al. Nature 2013; 496: 518-22
}

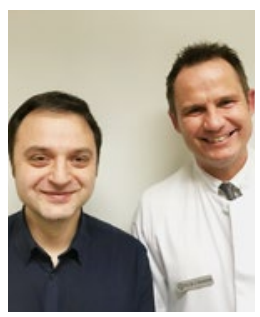

Dr. med. Refik Pul und

Prof. Dr. med. Christoph Kleinschnitz

Universitätsklinikum Essen, Klinik für Neurologie. E-Mail: refik.pul@uk-essen.de E-Mail: christoph.kleinschnitz@uk-essen.de 